\title{
Invasive lobular carcinoma co-existing with benign phylloides tumor
}

\author{
Shrestha $\mathrm{S}^{1}$, Sigdel B ${ }^{1}$, Pande $\mathrm{K}^{1}$, Gurung B \\ ${ }^{I}$ Department of Pathology, Patan Academy of Health Sciences, Patan Hospital, Kathmandu, Nepal.
}

\section{Keywords:}

Phylloides Tumor; Invasive Lobular carcinoma;

Estrogen receptor;

Progesterone receptor.

\begin{abstract}
Phylloides tumor constitutes less than $1 \%$ of all breast tumors and $2-3 \%$ of fibroepithelial breast tumors. Several histological parameters should be evaluated, including stromal cellularity, atypia, mitoses, stromal overgrowth, infiltrative borders, and presence or absence of necrosis. Here we report a case of a 60 years- old female who presented with left breast lump. Fine needle aspiration cytology was done which suggested epithelial hyperplasia with fibrocystic changes. Biopsy was performed which showed predominance of stromal hypercellularity with proliferation of spindle cells (no atypia, mitosis and stromal overgrowth were noticed). However, a focus showed proliferation of discohesive tumor cells arranged singly and in single file. A diagnosis of benign phylloides tumor with foci of invasive lobular carcinoma was made. The diagnosis was confirmed with IHC which showed intense $80 \%$ positivity for estrogen and progesterone receptor and spindle cells showing positivity for bcl-2. In situ lobular carcinoma component was not observed.
\end{abstract}

\section{INTRODUCTION}

Phylloides tumors are distinctly uncommon lesions in the female breast, constituting less than $1 \%$ of all breast tumors and 2 - $3 \%$ of fibroepithelial breast tumors. ${ }^{1}$ No single feature is reliable in predicting clinical behavior of phylloides tumors. Several histological parameters should be evaluated, including stromal cellularity, atypia, mitoses, stromal overgrowth, infiltrative borders, and presence or absence of necrosis. A mitotic rate of less than 5/10 high power field (HPF) suggests benign behavior, while a mitotic figure rate of $>10 / 10 \mathrm{HPF}$ suggests malignant potential. Carcinoma

\section{Correspondence:}

\section{Dr. Sabina Shrestha, MD}

Department of Pathology, Patan Academy of Health Sciences, Patan Hospital, Lalitpur, Nepal

Email: sabinapatho@gmail.com arising within a phylloides tumor is decidedly rare, with less than 30 reported cases in the literature. ${ }^{2}$ The concomitance of phylloides tumors with epithelial malignant neoplasms is rare. The literature reported the association of phylloides tumors with malignant epithelial components mainly in the form of ductal or lobular in situ lesions and less often in the invasive form. However, they are usually situated inside the lesion or near the fibroepithelial neoplasm tissue. ${ }^{3}$ Here we report a case of phylloides tumor with coexixtent lobular carcinoma with is extremely rare. ${ }^{4}$

\section{CASE REPORT}

A 60 years-old-female presented with left breast lump to surgical OPD, Patan hospital. The lump was firm, painless, and not adherent to skin without nipple retraction. FNAC done was suggestive of epithelial hyperplasia with fibrocystic 


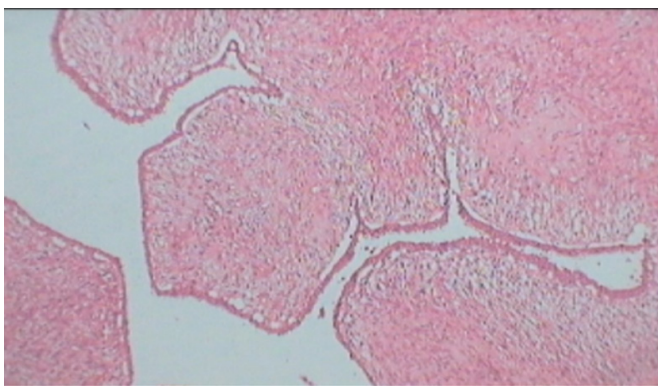

Figure 1a: Benign phylloides tumor (HE stain, X40).

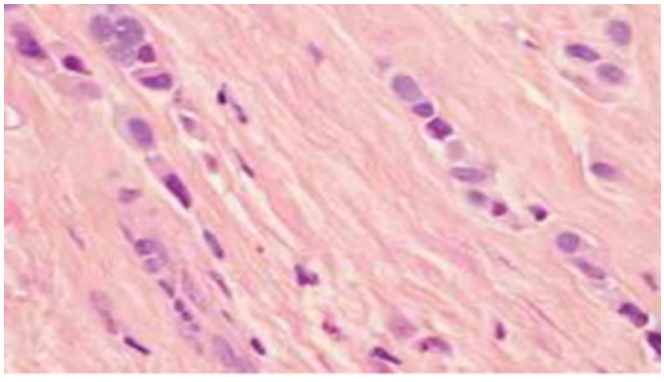

Figure 2: Photomicrograph showing Indian file pattern in lobular carcinoma (HE stain, X400).

changes. Mammography showed radiodense lesion replacing whole of the left breast with multiple lobulations and septation along with pleomorphic macrocalcifications hence was suspicious for malignancy. So, lumpectomy was performed.

Grossly, a bosselated nodule measuring $15 \times 13 \times 8 \mathrm{~cm}$ was received. Cut surface was solid and had myxoid appearance. $\mathrm{H} \& \mathrm{E}$ stained sections (fig.1a) showed predominantly stromal hypercellularity with proliferation of spindle cells (no atypia, mitosis and stromal overgrowth were noticed). However, one focus showed proliferation of discohesive tumor cells arranged singly and in single file. A diagnosis of benign phylloides tumor with foci of invasive lobular carcinoma (fig.2a) was made. The diagnosis was confirmed with IHC which showed intense $80 \%$ positivity for Estrogen (fig.2b) and progesterone receptor and spindle cells showing positivity for bcl-2 (fig. 1b). In situ lobular carcinoma component was not observed.

She subsequently underwent left radical mastectomy with axillary dissection. Grossly, mastectomy specimen with axillary tail measuring $10 \mathrm{x} 10 \mathrm{~cm}$ was received. Cut section showed fibrosed areas over previous scar marks. Solid areas were not identified grossly. Eleven lymph nodes were dissected out from axillary tail. Histopathology report was negative for residual tumor in breast and lymph nodes.

\section{DISCUSSION}

The epithelial component of phylloides tumors (whether

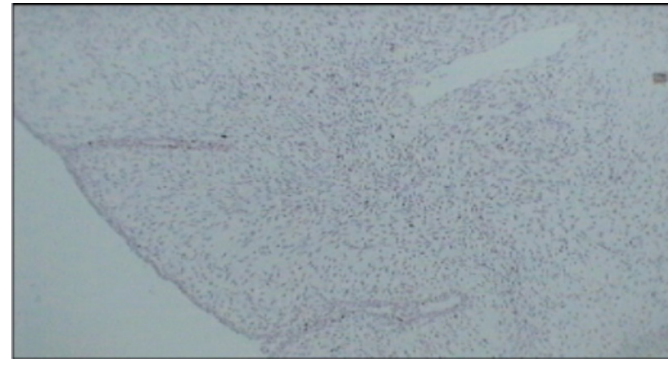

Figure 1b: Spindle cells positive for bcl-2 (IHC, X40).

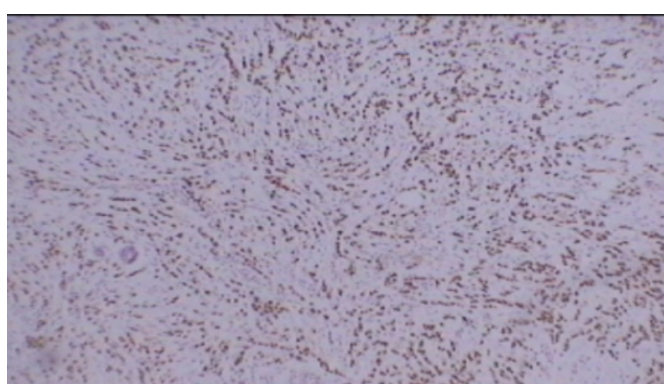

Figure 2b: Photomicrograph showing ER positivity in lobular carcinoma (IHC, X40). benign or malignant) may show a range of metaplastic (apocrine, squamous) and proliferative changes. ${ }^{5,6}$ The coexistent carcinoma and phylloides tumor is controversial. Some authors consider that the disease is due to a sudden transformation of the hyperplastic epithelium of the phylloides tumor, and others state that the carcinoma is caused haphazardly in the mammary gland adjunct to the phylloides tumor. This is due to the fact that the phylloides tumor usually takes up a larger area than the carcinoma. ${ }^{6}$ The age of the previously reported patients with coexistent carcinoma and phylloides tumor ranged from $31-80$ years, with most of the patients in the 5 th or 6 th decades ${ }^{7,8}$.

Even though carcinoma arising within phylloides tumor is rare, most cases are lobular type. The other reported subtypes include in situ and invasive ductal (no special type) carcinoma, tubular carcinoma and Squamous cell carcinoma. ${ }^{9}$

\section{CONCLUSION}

The present case emphasizes the fact that phylloides tumor may harbor intraductal and invasive carcinoma. Although the stroma in these tumors is commonly the aggressive component, the epithelial component also requires close histological appraisal. Besides the stromal component, the epithelial component of phylloides tumors should also be examined in detail, as it may harbor an in situ or invasive carcinoma. Treatment must be performed according to the carcinomatous component independently of the phylloides tumor 


\section{REFERENCES}

1. Trabelsia A, Abdelkrima SB, Stitaa W, Boudaggab MZ, Hammedia F, Mokn M .In Situ and Invasive Ductal Carcinoma Within a Borderline Phylloides Tumor. World J Oncol 2010;1:42-4.

2. JR, Armstrong C, O'Malley F, Ross J, and Tuck AB.In-situ and invasive carcinoma within a phylloides tumor associated with lymph node metastases. World J Surg Oncol 2004;15;2:46.

3. Neto GB, Rossetti C, Souza NA et al. Coexistence of benign phylloides tumor and invasive ductal carcinoma in distinct breasts: case report. European Journal of Medical Research 2012;17:8. CrossRef

4. Ishida T, Izuo M, Kawai T.Breast carcinoma arising in cystosarcoma phylloides: report of a case with a review of the literature. Jpn J Clin Oncol. 1984 Mar;14:99-106.
5. Leong ASY, Meredith DJ. Tubular carcinoma developing within a recurring cystosarcoma phylloides of the breast. Cancer. 1980;46:1863-7. CrossRef

6. Deodhar KK, Baraniya JB, Naresh KN,Shinde SR, Chinoy RF. Cancerization of phylloides tumor. Histopathology.1997;30:98-9. CrossRef

7. Knudsen PJT, Ostergaard J. Cystosarcoma phylloides with lobular and ductal carcinoma in situ. Arch Pathol Lab Med. 1987;111:87387

8. Nishimura R, Takahiro H, Imoto S, Mukai K. Malignant phylloides tumor with a noninvasive ductal carcinoma component. Virchows Arch. 1998;432:89-93. CrossRef

9. Guerino BN, Claudia R, Natalia AS et al. Coexistence of benign phylloides tumor and invasive ductal carcinoma in distinct breasts: case report. European Journal of Medical Research 2012:17:8. CrossRef 